PAPER

\title{
Should patients participate in clinical decision making? An optimised balance block design controlled study of goal setting in a rehabilitation unit
}

\author{
Rosaline C Holliday, Stefan Cano, Jennifer A Freeman, E Diane Playford
}

J Neurol Neurosurg Psychiatry 2007;78:576-580. doi: 10.1136/jnnp.2006.102509

See end of article for authors' affiliations

Correspondence to Dr E D Playford, Box 113, National Hospital for Neurology and Neurosurgery, Queen Square, London WCIN 3BG, UK; D.Playford@ ion.ucl.ac.uk

Received 17 July 2006 Revised 22 November 2006 Accepted 23November 2006 Published Online First 23 January 2007
Objectives: The recent National Service Framework for Long Term Conditions recommends that patients participate more in decision making about their care. However, few protocols exist to support this. One potentially useful method is goal setting, but little has been done to evaluate the added value of increasing patient participation in this way. Therefore, this study examined the impact of an increased participation goal setting protocol in a neurorehabilitation setting.

Design: The study was an $A B$ optimised balance block design with each block lasting 3 months, over an 18 month period.

Setting and participants: Patients $(n=201)$ were recruited from an inpatient neurological rehabilitation unit. Interventions: Patients $(n=100)$ recruited in phase A were involved in "usual practice" goal setting. Patients $(n=101)$ recruited in phase B were involved in "increased participation" goal setting, which included a protocol to help them define and prioritise their own goals.

Main outcome measures: Patients' perceptions of the relevance of goal setting and their autonomy within the process; the number, type and outcome of goals; and level of functional ability.

Results: Phase B patients ("increased participation") set fewer goals, of which significantly more were participation related. These patients perceived the goals to be more relevant, and expressed greater autonomy and satisfaction with goal setting. There were no differences in functional outcomes between the groups.

Conclusion: This study has shown that patients prefer increased participation in the goal setting process over standard procedures, perceiving their goals as more relevant and rehabilitation more patient centred despite the absence of functional gains. Effective patient centred care can be realised by using structures that help support patients to identify and communicate their priorities. As such, our findings suggest patients would benefit from greater participation in this aspect of clinical decision making.
A present, health care professionals tend to dominate consultations. ${ }^{1}$ It is increasingly acknowledged that a patient centred approach is required, which encourages patients to participate in and share control of treatment and management decisions, that take into account individual preferences within social contexts. ${ }^{2}$ This has been identified as a core requirement of the recently published National Service Framework for Long Term Conditions. ${ }^{3}$

The National Service Framework for Long Term Conditions aims to support individuals with long term conditions to selfmanage, maintain independence and achieve the best possible quality of life. ${ }^{3}$ In this context, care plans need to be agreed, developed and reviewed jointly by the individual and multidisciplinary healthcare team. One aspect of this process is to identify the individuals' goals. Goal setting is commonly used in rehabilitation, ${ }^{4}$ and other specialities that manage chronic disease use similar approaches. ${ }^{56}$

To date, little research has been done to evaluate the importance of participation in goal setting in a clinical environment. Studies in an occupational setting suggest that involving individuals in goal setting improves outcomes ${ }^{7}$ and increases knowledge. ${ }^{8}$ However, to be considered a useful part of the clinical management process, the added value of patient participation in goal setting should be demonstrated. This study examined the impact of a protocol to increase patient participation in goal setting that emphasised identification, discussion and negotiation of goals with health care professionals.

\section{METHODS}

\section{Design}

An optimised balance block design controlled study of goal setting was selected with each block lasting 3 months. The study was not blinded because it was not possible for patients, therapists or the researcher collecting outcome data $(\mathrm{RH})$ to be blinded to the nature of the goal setting process.

\section{Setting}

The Neurological Rehabilitation Unit at the National Hospital for Neurology and Neurosurgery, London, UK, is an 18 bed unit specialising in rehabilitation of patients with predominantly physical neurological deficits. The rehabilitation process implemented on the unit conforms with that described in the British Society of Rehabilitation Medicine clinical standards for specialist rehabilitation. ${ }^{9}$ Patients are referred from within the hospital, and from general practitioners and consultants from surrounding district and teaching hospitals. On average, 160 patients are admitted each year. Approximately 30\% of patients have sustained stroke, $30 \%$ have multiple sclerosis and 30\% have non-traumatic spinal cord lesions. The remaining group is made up of other neurological diagnoses, including central nervous system tumours and peripheral nerve disease. ${ }^{10}$ All patients are managed using a care pathway which incorporates a method of describing the body function, activity or

Abbreviations: FIM, Functional Independence Measure; GHQ-28, General Health Questionnaire 
Prior to admission

Initial assessment before admission by multidisciplinary team main areas to be addressed agreed by patient and team

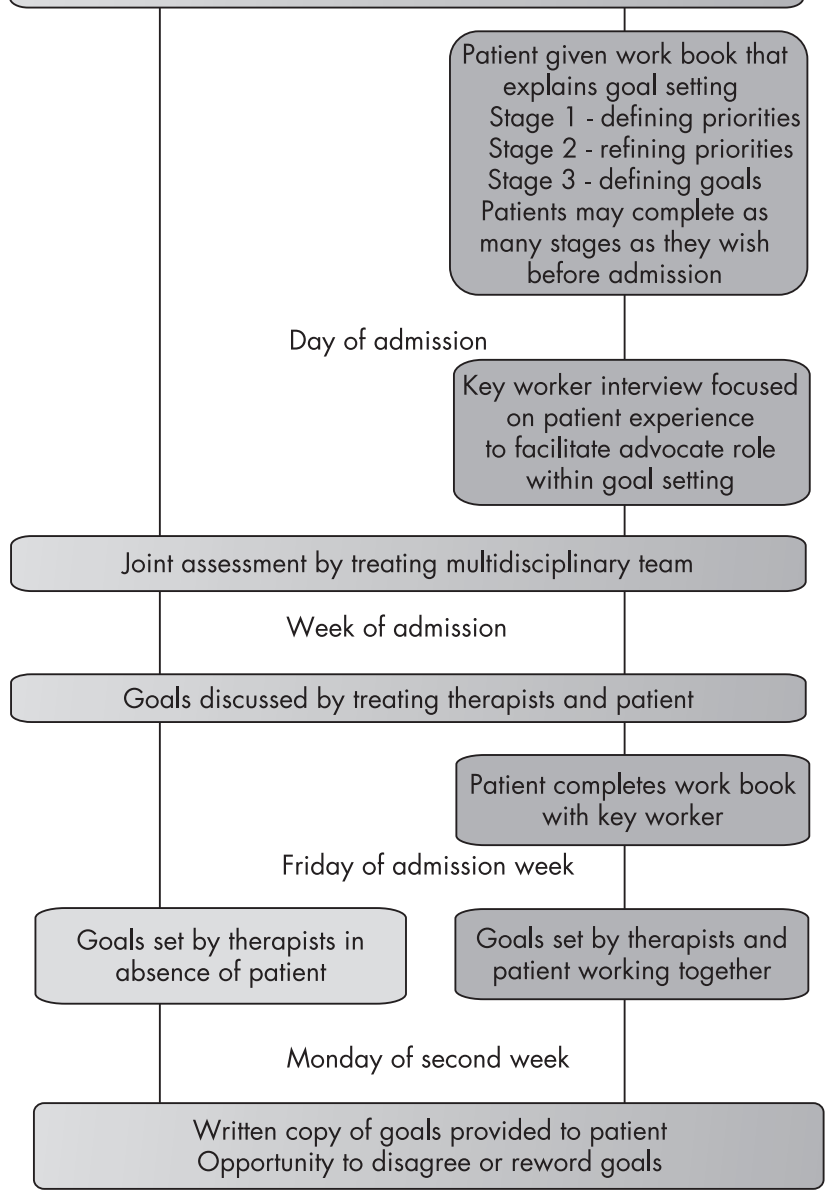

Figure 1 Flow diagram showing differences between group A (light grey) and group B (dark grey).

participation role addressed by each goal. Each goal is described by using up to four of 26 different goal components which are then grouped under the following five areas: (1) health maintenance, (2) cognitive functioning, (3) personal activities of daily living, (4) participation and (5) communication. Goals are described using up to four different components. The care pathway also lists reasons for failure to achieve goals, described as "goal variances". ${ }^{11}$

\section{Participants}

All patients admitted to the rehabilitation unit over an 18 month period were recruited to the study provided they had functional communication in English. Only five patients were excluded, two because of no spoken English and three because of severe dysphasia.

The study was powered using Functional Independence Measure (FIM) scores from previous studies. ${ }^{12}$ We calculated that 100 patients in each arm would enable us to detect a significant difference in functional outcome at the $\mathrm{p}<0.05$ level. There were no data available to power the study using the Payton participation scale or scales measuring goal relevance.

The study was approved by the Joint Research Ethics Committee of the National Hospital for Neurology and Neurosurgery and the Institute of Neurology. All patients provided informed consent, with the consent form specifying very clearly the differences in the two forms of goal setting under study.

\section{Intervention}

Two different approaches to goal setting were used in the study; the key features of these two methods are described in fig 1. The critical differences between the two approaches were the use of a "goal setting work book" completed by the patient and the presence of the patient in the goal setting meetings. The work book was in three sections. Patients were encouraged to work through the booklet initially with support from family and friends and then, if necessary, with support from their keyworker. The first section asked patients to prioritise activity and participation domains, and the second to identify specific tasks within those domains that they wished to work on. The final section involved determining what individuals wanted to achieve within the time frame of the rehabilitation admission.

The goal setting meeting provided a formal opportunity for therapists to discuss with patients both the projected outcome and the reasons for this. Patients could then set realistic goals. Both approaches resulted in a long term goal which specified the patients level of performance at discharge associated with a predicted date for discharge and short term goals, as "stepping stones" to the long term goal. These short term goals were reset on two or three weekly cycles depending on the duration of stay.

\section{Procedure}

A repetitive block design was selected with each block lasting 3 months. Three months was chosen for the duration of each block because analysis of admissions from the previous 3 years suggested that each block would then be balanced in terms of demographics and diagnosis. At the onset of each phase all staff (physiotherapists, occupational therapists, speech and language therapists, nurses and doctors) working on the neurological rehabilitation unit attended a training session on either the "usual practice" (phase A) or the "increased participation" (phase B) approach which was to be used. This minimised the confounding effects of newly acquired skills influencing the "usual practice" group, and controlled for any effect of teaching about goal setting and any potentially biasing attention being paid to a particular form of goal setting.

\section{Outcome measures}

A number of outcomes were measured:

(1) Patients' beliefs about their involvement in the goal setting process were measured on a four point patient autonomy scale based on the Patient Participation Scale. ${ }^{13 *}$

(2) Goal relevance was measured in two ways. Firstly, at discharge, patients were asked to provide a global assessment of the relevance of their goals using a $10 \mathrm{~cm}$ visual analogue scale with one end marked "goals highly relevant" and the other marked "goals of no relevance what so ever". ${ }^{14}$ In addition, patients were asked to categorise all of their goals into one of five groups: (1) highly relevant, (2) moderately relevant, (3) some relevance, (4) of little relevance and (5) of no relevance whatsoever. ${ }^{15}$

(3) Patient's overall satisfaction with the rehabilitation process was measured using a $10 \mathrm{~cm}$ visual analogue scale. ${ }^{14}$

"This scale describes the amount of "participation" an individual has in decision making and may more accurately be described as an autonomy scale. Throughout the rest of the paper the word participation is used as suggested by the World Health Organisation International Classification of function to mean engagement with social roles. 
Table 1 Comparison of goal numbers, goal categories and goal relevance between the "usual practice" and "increased participation" groups

\begin{tabular}{|c|c|c|c|}
\hline & $\begin{array}{l}\text { Phase A ("'usual } \\
\text { practice") }\end{array}$ & $\begin{array}{l}\text { Phase B ("increased } \\
\text { participation") }\end{array}$ & p Value \\
\hline Perception of participation $†$ & $3.3(0.64)[1-4]$ & $2.0(1.0)[1-4]$ & 0.001 \\
\hline Global goal relevance (VAS)† & 7.8 (1.7) [3-10] & 9.1 (1.1) [8-10] & 0.001 \\
\hline \multicolumn{4}{|c|}{ Mean individual goal relevance using "Likert scale" (mean (range)) } \\
\hline Proportion of high goal relevance & $65(23-100)$ & $80(20-100)$ & 0.001 \\
\hline Proportion of moderate goal relevance & $23(0-67)$ & $15(0-60)$ & 0.001 \\
\hline Proportion of some goal relevance & $7(0-27)$ & $4(0-40)$ & 0.001 \\
\hline Proportion of little goal relevance & $4(0-25)$ & $1(0-25)$ & 0.001 \\
\hline \multirow{2}{*}{\multicolumn{4}{|c|}{ Distribution of goal components (\%) }} \\
\hline & & & \\
\hline Health maintenance & 42 & 39 & $0.03^{*}$ \\
\hline Cognitive & 8 & 7 & $0.87^{*}$ \\
\hline PADL & 35 & 30 & $0.06^{*}$ \\
\hline Participation & 13 & 23 & $0.001^{*}$ \\
\hline Communication & 2 & 1 & $0.33^{*}$ \\
\hline \multicolumn{4}{|l|}{ Goal outcomes } \\
\hline No of goals per patient† & $17.9(11.2)[0-50]$ & $15(9.3)[0-45]$ & 0.04 \\
\hline Proportion of goals achieved (mean $\%$, range) & $81(11-100)$ & $83(40-100)$ & 0.38 \\
\hline Proportion of goals not achieved (mean $\%$, range) & $14(0-89)$ & $12(0-63)$ & 0.26 \\
\hline
\end{tabular}

(4) The distribution of the goal components was recorded, as were the outcome or end status of the goals, and reasons for non-completion of goals (variances) were collected for comparative purposes. ${ }^{11}$ Duration of stay in days was recorded to see if the different approaches resulted in shorter or longer durations of stay.

(5) Physical, social and psychological functional outcomes were measured on admission and discharge from the unit using the following well established validated rating scales:

- Functional Independence Measure (FIM). ${ }^{16}$

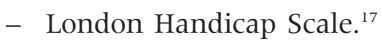

- General Health Questionnaire (GHQ-28). ${ }^{18}$

Finally, basic demographic data were collected for all patients including age, sex, ethnic background and diagnosis.

\section{Statistical analysis}

We hypothesised that increasing an individual's participation in goal setting during inpatient rehabilitation would result in goals that were perceived as more relevant to the individual and associated with greater satisfaction with the delivery of care. We did not anticipate any impact on function, as measured

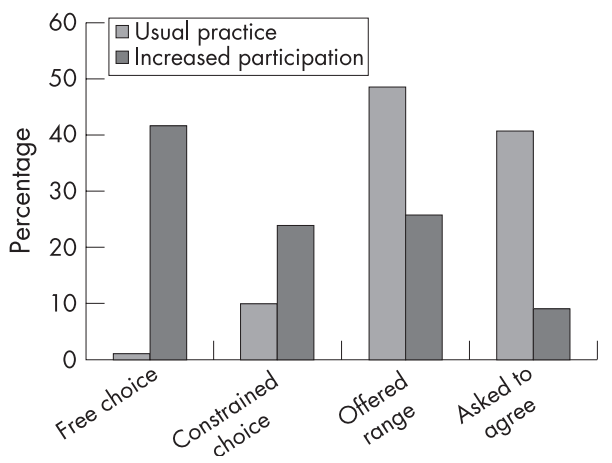

Figure 2 Comparison of reported extent of choice by group A ("usual practice") and group B ("increased participation"). using disability scales, as the intervention time was similar in both groups.

Scores were examined to compare phase A ("usual practice") and phase B ("increased participation"). Mean scores were compared using Student $t$ tests. Mann-Whitney tests were used on all other data, as appropriate. Data were analysed using the Statistics Package for Social Sciences (SPSS for Windows 12.0, 2005; SPSS Inc, Chicago, Illinois, USA).

\section{RESULTS}

\section{Patient characteristics}

The case mix, age, sex distribution and functional status of patients on admission and discharge were comparable with other studies reported from this unit. ${ }^{10}$

There were no significant differences between the phase A ("usual practice") and phase B ("increased participation") groups in terms of sex, ethnic origin or case mix. However, mean age for the "increased participation" group was 4 years younger than the "usual practice" group $(\mathrm{p}<0.05$; data available from the authors).

\section{Patient beliefs about extent of choice (fig 2)}

The majority of the phase B ("increased participation") group reported that they were able to choose their own goals, whereas the majority of the phase A ("usual practice") group reported

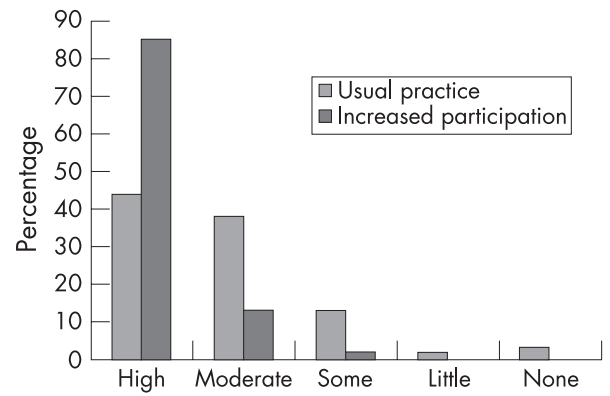

Figure 3 Comparison of goal relevance between group A ("usual practice") and group B ("increased participation"). 
that they were asked to agree to team formulated goals $(\mathrm{p}<0.001$; data available from the authors).

\section{Goal relevance (table 1, fig 3)}

The global assessment of goal relevance was significantly higher in the phase B ("increased participation") group $(\mathrm{p}<0.001)$. Also, the proportion of goals perceived as highly relevant was also higher in group B $(\mathrm{p}<0.001)$

\section{Patient satisfaction}

Patients in the phase B ("increased participation") group reported significantly higher satisfaction with the rehabilitation process $(\mathrm{p}<0.001$; data available from the authors). Only one patient questioned their group allocation, and was offered a chance to withdraw from the study. They chose to continue within the study in their allocated phase A ("usual practice") group.

\section{Goal components (table 1)}

The spread of goals differed between the groups with a significantly higher proportion of participation related goals being set in the "increased participation" group.

\section{Goal outcomes}

There were significantly fewer goals set in the phase B group $(p=0.04)$. The proportion of goals achieved was not different between the groups $(\mathrm{p}=0.38)$.

\section{Functional outcomes}

There were no differences between groups in functional outcomes on admission or discharge. Following rehabilitation, all patients demonstrated a significant improvement in all measures similar to those reported in previous studies from this unit (data available from the authors). There were no significant differences in the change scores for these measures with the exception of the GHQ-28 which showed the phase B ("increased participation") group making smaller gains $(\mathrm{p}=0.037$; data available from the authors $)$. The two groups did not differ in duration of stay.

\section{DISCUSSION}

This study has shown that providing individuals who have long term neurological conditions with a structure that allowed them to clearly define and articulate their goals resulted in greater perceived autonomy, and greater perceived relevance of goals. In addition, rehabilitation appeared more precisely targeted. Although fewer goals were set, those goals were centred around the individual's participation in life roles, associated with higher relevance.

Defining structures that help individuals articulate their ambitions both "support the person, their family and carers to contribute to planning the rehabilitation process" and enable professionals to work with those individuals in a way that is "focused on individual goals beyond basic daily care and promote participation in a full range of life roles", as recommended in the National Service Framework. ${ }^{1}$ Current consensus views suggest that delivering care in a manner that is meaningful for the individual is more likely to lead to long term "adherence" or, in the case of inpatient rehabilitation, result in the transfer of newly learnt skills to the individual's own home and community.

This is the largest published controlled study of goal setting. Although the gold standard for clinical trials is the randomised controlled trial, we felt this was not an appropriate design for this study because the new skills identified in the "increased participation" arm would unavoidably contaminate the "usual practice" (phase A) arm. The lack of blinding is also a potential source of bias, although this was minimised through the use of self-report outcomes. Blinding the participants was not possible for practical reasons. During each block participants were all treated in the same way and only one participant queried his group allocation. Thus participants did not appear unhappy with either form of goal setting.

The patients in this study are typical of many patients seen by both inpatient and outpatient rehabilitation services. However, our patients had relatively mild cognitive deficits which may not be applicable to all patients. Despite this, previous research suggests that even patients with significant cognitive impairments can set realistic goals. ${ }^{19} 20$ The age differences between the two arms also needs to be considered, although given the age range of the patients who participated in the study this is unlikely to be of clinical significance.

As in previous studies, all patients showed improvements in outcomes, measured using the FIM, London Handicap Scale and GHQ-28. Although we found no differences in functional outcomes between the two groups, there was a significance difference in GHQ change scores. In the past we have shown that although physical gains are maintained, emotional gains are not. ${ }^{21}$ It is possible that the increased participation group remain focussed on the difficulties they experience outside hospital, so that although their emotional gains are smaller they may be more robust. This requires further study.

Patient centred care involves the development of new skills and attitudes. Much of the previous literature has focussed on fostering attitudes that allow clinicians to develop skills as facilitators. In addition, there has been recent work that acknowledges the role of the patient as "expert". ${ }^{22}$ However, to our knowledge, this is the first study to demonstrate that the use of a specific protocol to support patients in the identification and communication of their priorities results in changes in the focus of rehabilitation interventions. This change appears to support individuals in maintaining both activity and participation, and may be important in promoting self-management and well being. Developing structures and processes that support effective partnerships is an important area for further study.

Goal setting is a term used almost uniquely in rehabilitation but the task described by goal setting is performed by a wide range of health care disciplines working with individuals with long term conditions, for example diabetes and arthritis. ${ }^{56}$ The approaches used in this study may have wider applicability than patients with neurological disability. Future studies should focus on extending the range of conditions managed using this approach.

\section{ACKNOWLEDGEMENTS}

The authors would like to thank all the staff and patients on the Neurological Rehabilitation Unit who participated in this study.

\section{Authors' affiliations}

Rosaline C Holliday, Stefan Cano, Jennifer A Freeman, E Diane Playford, Institute of Neurology, University College London, London, UK Jennifer A Freeman, Faculty of Health and Social Work, Plymouth University, Devon, UK

The study was funded by the UCLH Clinical Research and Development Committee.

\section{Competing interests: None}

Dr Diane Playford conceived the study and accepts full responsibility for the conduct of the study, had access to the data and controlled the decision to publish. Ms Ros Holliday coordinated the project and collected, analysed and interpreted the data, and drafted the first version of the paper. Dr Jennifer Freeman and Dr Stefan Cano contributed to supervising the data analysis and interpreting the data and revised the paper critically. All authors contributed to the writing of the paper and approved the final version to be published. 


\section{REFERENCES}

1 Berry DL, Wilkie DJ, Thomas CR Jr, et al. Clinicians communicating with patients experiencing cancer pain. Cancer Invest 2003;21:374-81.

2 Lewin SA, Skea ZC, Entwistle V, et al. Interventions for providers to promote a patient-centred approach in clinical consultations. Cochrane Database Systemic Review. Chichester: Wiley Interscience, 2001;4, CD003267.

3 The National Service Framework for Long Term Conditions DoH. March 2005. http://www.dh.gov.uk/PublicationsAndStatistics/Publications/ PublicationsPolicyAndGuidance/PublicationsPolicyAndGuidanceArticle/fs/ en?CONTENT ID = 4105361 \&chk = il7dri (accessed 28 March 2007).

4 Wade DT. Evidence relating to goal planning in rehabilitation. Clin Rehabil 1998;12:273-5.

5 Osborne RH, Spinks JM, Wicks IP Patient education and self-management programs in arthritis. Med J Aust 2004;180(5 Suppl):S23-6.

6 Deakin T, McShane CE, Cade JE, et al. Group based training for self management strategies in people with type 2 diabetes mellitus. Cochrane Database Systematic Review. Chichester: Wiley Interscience, 2005; 18(2):CD003417.

7 Latham GP, Mitchell TR, Dossett DL. The importance of participative goal setting and anticipated rewards on goal difficulty and job performance. J Appl Psychol 1978;63: 163-71

8 Locke EA, Latham GP. Building a practically useful theory of goal setting and task motivation. A 35-year odyssey. Am Psychol 2002;57:705-17.

9 Turner-Stokes L, Williams H, Abraham R, et al. Clinical standards for inpatient specialist rehabilitation services in the UK. Clin Rehabil 2000;14:468-80.

10 Freeman JA, Hobart JC, Playford ED, et al. Evaluating neurorehabilitation: lessons from routine data collection. I Neurol Neurosurg Psychiatry 2005;76:723-8.
11 Edwards SG, Thompson AJ, Playford ED. Integrated care pathways: diseasespecific or process-specific, Clin Med 2004;4:132-5.

12 Freeman JA, Langdon DW, Hobart JC, et al. The impact of inpatient rehabilitation on progressive multiple sclerosis. Ann Neurol 1997;42:236-44.

13 Payton OD, Nelson CE, Ozer MN. Patient participation in program planning: a manual for therapists. Philadelphia: FA Davis Company, 1990.

14 Indredavik B, Bakke F, Slordahl SA, et al. Stroke unit treatment improves longterm quality of life: a randomized controlled trial. Stroke 1998;29:895-9.

15 Pell G. Use and misuse of Likert scales. Med Educ 2005;39:970.

16 Dodds TA, Martin DP, Stolov WC, et al. A validation of the functional independence measurement and its performance among rehabilitation inpatients. Arch Phys Med Rehabil 1993;74:531.

17 Harwood RH, Rogers A, Dickinson E, et al. Measuring handicap: the London Handicap Scale, a new outcome measure for chronic disease. Qual Health Care 1994:3:11-16.

18 Goldberg DP, Hillier VF. A scaled version of the General Health Questionnaire. Psychol Med 1979;9:139-45.

19 Trombly CA, Radomski MV, Trexel C, et al. Occupational therapy and achievement of self-identified goals by adults with acquired brain injury: phase II. Am J Occup Ther 2002;56:489-98.

20 Gauggel S, Hoop M, Werner K. Assigned versus self set goals and their impact on the performance of brain-damaged patients. J Clin Exp Neuropsychol 2002;24:1070-80.

21 O'Connor RJ, Cano SJ, Thompson AJ, et al. The impact of inpatient neurorehabilitation on psychological well-being on discharge and at 3 month follow-up. J Neurol 2005;252:814-19.

22 Lorig K, Sobel DS, Stewart AL, et al. Evidence suggesting that a chronic disease self-management program can improve health status while reducing hospitalization. A randomized trial. Med Care 1999;37:5-14.

\section{bmjupdates+}

bmjupdates+ is a unique and free alerting service, designed to keep you up to date with the medical literature that is truly important to your practice.

bmjupdates+ will alert you to important new research and will provide you with the best new evidence concerning important advances in health care, tailored to your medical interests and time demands.

Where does the information come from?

bmiupdates+ applies an expert critical appraisal filter to over 100 top medical journals A panel of over 2000 physicians find the few 'must read' studies for each area of clinical interest

Sign up to receive your tailored email alerts, searching access and more...

www.bmjupdates.com 